\title{
Twisted Alexander polynomials and a partial order on the set of prime knots
}

\author{
TERUAKI KITANO \\ MASAAKI SUZUKI
}

\begin{abstract}
We give a survey of some recent papers by the authors and Masaaki Wada $[6 ; 7 ; 8]$ relating the twisted Alexander polynomial with a partial order on the set of prime knots. We also give examples and pose open problems.
\end{abstract}

57M25; 57M05

Dedicated to Fred Cohen on the occasion of his 60th birthday

\section{Introduction}

The twisted Alexander polynomial was introduced by Lin [9] and Wada [18] independently. Lin defined it for a knot by using a regular Seifert surface and Wada defined it for a finitely presentable group by using Fox's free differential calculus. More generally, Jiang and Wang [3] studied twisted topological invariants for a 3-manifold by using representations of the fundamental group. Following this work, much research from many viewpoints has been carried out (or is currently being carried out) related to this invariant. See the bibliography for more precise details.

In this paper, we give a survey of some recent papers by the authors and Masaaki Wada $[6 ; 7 ; 8]$ relating the twisted Alexander polynomial with a partial order on the set of prime knots. We also give examples and pose open problems.

In Section 2 we recall the definition of the twisted Alexander polynomial for a finitely presentable group as given by Wada. His definition is purely algebraic.

In Section 3 we state one of the fundamental results, which gives a relation between the existence of a surjection of groups and the twisted Alexander polynomial; see [8] for more details. This result gives us a new criterion for the non-existence of a surjective homomorphism between two groups. The corresponding fact about the (classical) Alexander polynomial is well known; see Crowell and Fox [1].

In Section 4 we explain through examples how to use twisted Alexander polynomials to determine the partial order on the set of prime knots defined by the existence of a 
surjective homomorphism between the knot groups. Using the result of Section 3, we determined in [6] which pairs of prime knots from Rolfsen's knot table [15] have this relation. Rolfsen's knot table lists all the prime knots of 10 crossings or less.

In Section 5, we consider some problems which arise naturally from the results of Section 4. The existence problem of a surjective homomorphism between knot groups, or more generally 3-manifold groups, is related with the theory of degree one maps and the period of a knot.

In Section 6 we pose further problems for future study.

\section{Definition of the twisted Alexander polynomial}

In this section, we briefly recall the algebraic definition of the twisted Alexander polynomial, by using Fox's free differential calculus.

Let $G$ be a finitely presentable group. Choose a presentation of $G$ :

$$
G=\left\langle x_{1}, \ldots, x_{u} \mid r_{1}, \ldots, r_{v}\right\rangle .
$$

Suppose $\alpha: G \rightarrow\left\langle t_{1}, \ldots, t_{l} \mid t_{i} t_{j}=t_{j} t_{i}\right\rangle \cong \mathbb{Z}^{l}$ is a surjective homomorphism to the free abelian group with generators $t_{1}, \ldots, t_{l}$ and $\rho: G \rightarrow G L(n ; R)$ a linear representation, where $R$ is a unique factorization domain. These maps naturally induce ring homomorphisms $\tilde{\rho}$ and $\widetilde{\alpha}$ from $\mathbb{Z}[G]$ to $\mathbb{Z}[G L(n ; R)]=M(n ; R)$ and $\mathbb{Z}\left[t_{1}{ }^{ \pm 1}, \ldots, t_{l}^{ \pm 1}\right]$ respectively, where $M(n ; R)$ denotes the matrix algebra of degree $n$ over $R$. Then $\tilde{\rho} \otimes \tilde{\alpha}$ defines a ring homomorphism

$$
\mathbb{Z}[G] \rightarrow M\left(n ; R\left[t_{1}^{ \pm 1}, \ldots, t_{l}^{ \pm 1}\right]\right) .
$$

Let $F_{u}$ be the free group on generators $x_{1}, \ldots, x_{u}$ and let

$$
\Phi: \mathbb{Z}\left[F_{u}\right] \rightarrow M\left(n ; R\left[t_{1}^{ \pm 1}, \ldots, t_{l}^{ \pm 1}\right]\right)
$$

be the composite of the surjection $\mathbb{Z}\left[F_{u}\right] \rightarrow \mathbb{Z}[G]$ induced by the fixed presentation and the map $\tilde{\rho} \otimes \tilde{\alpha}: \mathbb{Z}[G] \rightarrow M\left(n ; R\left[t_{1}^{ \pm 1}, \ldots, t_{l}^{ \pm 1}\right]\right)$.

We define the $v \times u$ matrix $M$ whose $(i, j)$ component is the $n \times n$ matrix

$$
\Phi\left(\frac{\partial r_{i}}{\partial x_{j}}\right) \in M\left(n ; R\left[t_{1}^{ \pm 1}, \ldots, t_{l}^{ \pm 1}\right]\right)
$$

where $\partial / \partial x$ denotes the Fox differential. This matrix $M$ is called the Alexander matrix of the fixed presentation of $G$ associated to the representation $\rho$. 
It is easy to see that there is an integer $1 \leq j \leq u$ such that $\operatorname{det} \Phi\left(x_{j}-1\right) \neq 0$. For such $j$, let us denote by $M_{j}$ the $v \times(u-1)$ matrix obtained from $M$ by removing the $j$-th column. We regard $M_{j}$ as an $n v \times n(u-1)$ matrix with coefficients in $R\left[t_{1}^{ \pm 1}, \ldots, t_{l}^{ \pm 1}\right]$. Moreover, for an $n(u-1)$-tuple of indices

$$
I=\left(i_{1}, i_{2}, \ldots, i_{n(u-1)}\right) \quad\left(1 \leq i_{1}<i_{2}<\cdots<i_{n(u-1)} \leq n v\right),
$$

we denote by $M_{j}^{I}$ the $n(u-1) \times n(u-1)$ square matrix consisting of the $i_{k}$-th rows of the matrix $M_{j}$, where $k=1,2, \ldots, n(u-1)$.

Then the twisted Alexander polynomial of a finitely presented group $G$ for a representation $\rho: G \rightarrow G L(n ; R)$ is defined to be a rational expression

$$
\Delta_{G, \rho}\left(t_{1}, \ldots, t_{l}\right)=\frac{\operatorname{gcd}_{I}\left(\operatorname{det} M_{j}^{I}\right)}{\operatorname{det} \Phi\left(x_{j}-1\right)} .
$$

Here $\operatorname{gcd}_{I}\left(\operatorname{det} M_{j}^{I}\right)$ is the greatest common divisor of $\left\{\operatorname{det} M_{j}^{I} \mid I \subset\{1, \ldots, n v\}\right\}$. It is shown that $\Delta_{G, \rho}\left(t_{1}, \ldots, t_{l}\right)$ is independent of the choice of $j$ such that $\operatorname{det} \Phi\left(x_{j}-1\right) \neq$ 0 up to a factor $\epsilon t_{1}{ }^{\varepsilon_{1}} \cdots t_{l}{ }^{\varepsilon_{l}}$, where $\epsilon \in R^{\times}, \varepsilon_{i} \in \mathbb{Z}$. Moreover, we can check that Tietze transformations on the presentation of $G$ do not affect the twisted Alexander polynomial. Therefore the twisted Alexander polynomial $\Delta_{G, \rho}\left(t_{1}, \ldots, t_{l}\right)$ is independent of the choice of the presentation of $G$. See Wada [18] for details here.

\section{Twisted Alexander polynomials and surjectivity of a group homomorphism}

In this section, we present the following theorem from [8], which is one of the fundamental theorems, for applications [6; 7] of twisted Alexander polynomials.

Theorem 3.1 Let $G$ and $G^{\prime}$ be finitely presentable groups and $\alpha, \alpha^{\prime}$ surjective homomorphisms from $G, G^{\prime}$ to $\mathbb{Z}^{l}$ respectively. Suppose that there exists a surjective homomorphism $\varphi: G \rightarrow G^{\prime}$ such that $\alpha=\alpha^{\prime} \circ \varphi$. Then $\Delta_{G, \rho}$ is divisible by $\Delta_{G^{\prime}, \rho^{\prime}}$ for any representation $\rho^{\prime}: G^{\prime} \rightarrow G L(n ; R)$, where $\rho=\rho^{\prime} \circ \varphi$. More precisely, the quotient of $\Delta_{G, \rho}$ by $\Delta_{G^{\prime}, \rho^{\prime}}$ is a Laurent polynomial in $R\left[t_{1}^{ \pm 1}, \ldots, t_{l}^{ \pm}\right]$.

The main motivation here is the following. Let $G(K)$ be the knot group $\pi_{1}\left(S^{3}-K\right)$ of a knot $K$ in $S^{3}$. For any knots $K, K^{\prime}$, if there exists a surjective homomorphism from $G(K)$ to $G\left(K^{\prime}\right)$, then the Alexander polynomial of $K$ is divisible by that of $K^{\prime}$. Murasugi [12] mentions that if there exists a surjective homomorphism from a knot group $G(K)$ to the trefoil knot group, then the twisted Alexander polynomial of $G(K)$ 
is divisible by that of the trefoil knot group. Theorem 3.1 is a generalization of his result.

Theorem 3.1 is proved by two different methods in [6]. The first one is purely algebraic using the definition of the twisted Alexander polynomial and linear algebra. The second one uses Reidemeister torsion. The twisted Alexander polynomial of a knot group may be interpreted in terms of Reidemeister torsion in the same way as the classical Alexander polynomial. These invariants of knots have been studied from the Reidemeister torsion viewpoint. For example, see Milnor [11], Kitano [5] or Kirk and Livingston [4].

We now consider an easy algebraic situation as an example.

Example 3.2 Let $G=\left\langle x_{1}, \ldots, x_{u} \mid r_{1}, \ldots, r_{v}\right\rangle$ be a finitely presented group with the abelianization $\alpha: G \rightarrow \mathbb{Z}^{l}$ and $\rho: G \rightarrow G L(n ; R)$ a representation of $G$. Another group $G^{\prime}$ is defined by

$$
G^{\prime}=\left\langle x_{1}, \ldots, x_{u} \mid r_{1}, \ldots, r_{v}, s\right\rangle
$$

where $s$ is a word of $x_{1}, \ldots, x_{u}$. The natural projection $\pi: G \rightarrow G^{\prime}$ is surjective. Suppose that the word $s$ belongs to $\operatorname{ker} \alpha$ and $\operatorname{ker} \rho$, then there exists a surjective homomorphism $\alpha^{\prime}: G^{\prime} \rightarrow \mathbb{Z}^{l}$ and a representation $\rho^{\prime}: G^{\prime} \rightarrow G L(n ; R)$ such that $\alpha=\alpha^{\prime} \circ \pi$ and $\rho=\rho^{\prime} \circ \pi$. By Theorem 3.1, $\Delta_{G, \rho}$ is divisible by $\Delta_{G^{\prime}, \rho^{\prime}}$. Here we will verify the divisibility by the definition. Let $M$ be the Alexander matrix of $G$ associated to $\rho$. Then the Alexander matrix $M^{\prime}$ of $G^{\prime}$ associated to $\rho^{\prime}$ is obtained by adding $n$ rows

$$
\left(\Phi\left(\frac{\partial s}{\partial x_{1}}\right) \Phi\left(\frac{\partial s}{\partial x_{2}}\right) \cdots \Phi\left(\frac{\partial s}{\partial x_{u}}\right)\right)
$$

to $M$. Since the numerator of $\Delta_{G^{\prime}, \rho^{\prime}}$ is the greatest common divisor of $\operatorname{det} M^{\prime I^{\prime}}$, $\operatorname{gcd}_{I^{\prime}}\left(\operatorname{det} M_{j}^{\prime I^{\prime}}\right)$ is a divisor of $\operatorname{gcd}_{I}\left(\operatorname{det} M_{j}^{I}\right)$. Furthermore, the denominators of $\Delta_{G, \rho}$ and $\Delta_{G^{\prime}, \rho^{\prime}}$ are the same. Hence $\Delta_{G, \rho}$ is divisible by $\Delta_{G^{\prime}, \rho^{\prime}}$.

\section{A partial order in the knot table}

In this section we consider prime knots. Let $K$ be a prime knot and $G(K)$ its knot group. A partial order on the set of prime knots is defined as follows. For two prime knots $K_{1}, K_{2}$, we write $K_{1} \geq K_{2}$ if there exists a surjective group homomorphism from $G\left(K_{1}\right)$ onto $G\left(K_{2}\right)$. It is known that the relation $\geq$ satisfies the condition of a partial order on the set of prime knots. The following result [6] can be obtained by applying Theorem 3.1 and using a computer. 
Theorem 4.1 The above partial order on the knots in Rolfsen's table is given as below:

$$
\begin{gathered}
8_{5}, 8_{10}, 8_{15}, 8_{18}, 8_{19}, 8_{20}, 8_{21}, 9_{1}, 9_{6}, 9_{16}, 9_{23}, 9_{24}, 9_{28}, 9_{40}, \\
10_{5}, 10_{9}, 10_{32}, 10_{40}, 10_{61}, 10_{62}, 10_{63}, 10_{64}, 10_{65}, 10_{66}, 10_{76}, 10_{77}, 10_{78}, \quad \geq 3_{1}, \\
10_{82}, 10_{84}, 10_{85}, 10_{87}, 10_{98}, 10_{99}, 10_{103}, 10_{106}, 10_{112}, 10_{114}, 10_{139}, 10_{140}, \quad \\
10_{141}, 10_{142}, 10_{143}, 10_{144}, 10_{159}, 10_{164} \\
8_{18}, 9_{37}, 9_{40}, 10_{58}, 10_{59}, 10_{60}, 10_{122}, 10_{136}, 10_{137}, 10_{138} \geq 4_{1}, \\
10_{74}, 10_{120}, 10_{122} \geq 5_{2} .
\end{gathered}
$$

First, we explain how to show the non-existence of surjective homomorphisms by using the twisted Alexander polynomials. Then, we construct explicitly a surjective homomorphism for each pair of knots which belongs to the above list.

\subsection{Non-existence of surjective homomorphisms}

We see how to apply the twisted Alexander polynomials of knots for the non-existence problem of surjective homomorphisms between knot groups.

For many pairs of knots which do not appear in Theorem 4.1, we can show easily that there exists no surjective homomorphism between their knot groups by using the classical Alexander polynomial. If the Alexander polynomial of $G(K)$ is not divisible by that of $G\left(K^{\prime}\right)$, then there exists no surjective homomorphism from $G(K)$ to $G\left(K^{\prime}\right)$. However, for some cases, it cannot be determined by using only the Alexander polynomial whether or not there exists a surjective homomorphism between knot groups.

Example 4.2 We explain how to find whether or not there exists a surjective homomorphism between $G\left(8_{11}\right)$ and $G\left(3_{1}\right)$. The classical Alexander polynomials of them are respectively

$$
\begin{aligned}
\Delta_{8_{11}} & =2 t^{4}-7 t^{3}+9 t^{2}-7 t+2, \\
\Delta_{3_{1}} & =t^{2}-t+1 .
\end{aligned}
$$

Because $\Delta_{8_{11}}$ does not divide $\Delta_{3_{1}}$ clearly, there exists no surjective homomorphism from $G\left(3_{1}\right)$ to $G\left(8_{11}\right)$. Therefore we can see

$$
3_{1} \nsucceq 8_{11} \text {. }
$$

However, since $\Delta_{3_{1}}$ divides $\Delta_{8_{11}}$, we cannot determine whether or not there exists a surjective homomorphism from $G\left(8_{11}\right)$ to $G\left(3_{1}\right)$. 
In cases that we can not determine the non-existence of a surjection, we apply the twisted Alexander polynomial to the knot groups.

We take a Wirtinger presentation of any knot group $G(K)$ as follows:

$$
G(K)=\left\langle x_{1}, x_{2}, \ldots, x_{u} \mid r_{1}, r_{2}, \ldots, r_{u-1}\right\rangle .
$$

Here we note that generators are conjugate with each other. The abelianization

$$
\alpha: G(K) \rightarrow \mathbb{Z} \cong\langle t\rangle
$$

is obtained by mapping each generator $x_{i}$ to $t$. We consider the twisted Alexander polynomial for a 2-dimensional unimodular representation over a finite field. Now we fix a prime integer $p$ and take a representation

$$
\rho: G(K) \rightarrow S L\left(2 ; \mathbb{F}_{p}\right) .
$$

Here $\mathbb{F}_{p}$ is the finite prime field $\mathbb{Z} / p \mathbb{Z}$. Then we obtain the Alexander matrix $M$ associated to $\rho$ in $M\left((u-1) \times u ; M\left(2 ; \mathbb{F}_{p}\left[t, t^{-1}\right]\right)\right)$. Further it always holds that $\operatorname{det} \Phi\left(x_{1}-1\right) \neq 0$ because

$$
\begin{aligned}
\Phi\left(x_{1}-1\right) & =\alpha\left(x_{1}\right) \otimes \rho\left(x_{1}\right)-E \\
& =t \rho\left(x_{1}\right)-E
\end{aligned}
$$

where $E$ is the identity matrix of degree 2 . We write $M_{1}$ for the $(u-1) \times(u-1)$ matrix obtained from $M$ by removing the first column. Here $\Delta_{K, \rho}^{N}(t)$ denotes the determinant of $M_{1}$ and $\Delta_{K, \rho}^{D}(t)$ the determinant of $\Phi\left(x_{1}-1\right)$.

In this case, the twisted Alexander polynomial of $G(K)$ for a representation $\rho: G(K) \rightarrow$ $\operatorname{SL}\left(2 ; \mathbb{F}_{p}\right)$ is defined to be

$$
\Delta_{K, \rho}(t)=\frac{\Delta_{K, \rho}^{N}(t)}{\Delta_{K, \rho}^{D}(t)}=\frac{\operatorname{det} M_{1}}{\operatorname{det} \Phi\left(x_{1}-1\right)} .
$$

Here we can prove the non-existence of a surjective homomorphism between the groups of any two knots except for the pairs listed in Theorem 4.1. A criterion for the non-existence is obtained by applying Theorem 3.1 for knot groups.

Corollary 4.3 Let $K_{1}$ and $K_{2}$ be two knots. If there exists a representation $\rho_{2}: G\left(K_{2}\right) \rightarrow S L\left(2 ; \mathbb{F}_{p}\right)$ such that $\Delta_{K_{1}, \rho_{1}}^{N}(t)$ is not divisible by $\Delta_{K_{2}, \rho_{2}}^{N}(t)$ or $\Delta_{K_{1}, \rho_{1}}^{D}(t) \neq \Delta_{K_{2}, \rho_{2}}^{D}(t)$ for any representation $\rho_{1}: G\left(K_{1}\right) \rightarrow S L\left(2 ; \mathbb{F}_{p}\right)$, then there exists no surjective homomorphism from $G\left(K_{1}\right)$ onto $G\left(K_{2}\right)$. 
By applying Corollary 4.3 with the aid of a computer, we can prove that there exists no surjective homomorphism between the remaining pairs of knots. All the twisted Alexander polynomials which we use to check the non-existence of surjections are listed in [6].

Example 4.4 We can show the non-existence of a surjective homomorphism from $G\left(8_{11}\right)$ to $G\left(3_{1}\right)$ by using the twisted Alexander polynomials of $S L\left(2 ; \mathbb{F}_{5}\right)$-representations as follows.

First, we compute that the numerators and denominators of the twisted Alexander polynomials of $G\left(8_{11}\right)$ associated to all $S L\left(2 ; \mathbb{F}_{5}\right)$-representations. We obtain the pairs of $\left(\Delta_{8_{11}, \rho_{i}}^{N}(t), \Delta_{8_{11}, \rho_{i}}^{D}(t)\right)(i=1,2, \ldots, 10)$ as follows:

$$
\begin{aligned}
& \left(\Delta_{811, \rho_{i}}^{N}(t), \Delta_{8_{11}, \rho_{i}}^{D}(t)\right)= \\
& \left(2 t^{6}+2 t^{5}+t^{4}+4 t^{3}+t^{2}+2 t+2, t^{2}+t+1\right), \\
& \left(3 t^{6}+2 t^{5}+4 t^{4}+4 t^{3}+4 t^{2}+2 t+3, t^{2}+4 t+1\right), \\
& \left(t^{8}+t^{6}+t^{2}+1, t^{2}+1\right), \\
& \left(2 t^{8}+2 t^{7}+4 t^{6}+t^{5}+4 t^{4}+t^{3}+4 t^{2}+2 t+2, t^{2}+4 t+1\right), \\
& \left(2 t^{8}+3 t^{7}+4 t^{6}+4 t^{5}+4 t^{4}+4 t^{3}+4 t^{2}+3 t+2, t^{2}+t+1\right), \\
& \left(4 t^{8}+3 t^{6}+t^{4}+3 t^{2}+4, t^{2}+1\right), \\
& \left(4 t^{8}+t^{7}+t^{6}+3 t^{4}+t^{2}+t+4, t^{2}+4 t+1\right), \\
& \left(4 t^{8}+2 t^{7}+t^{5}+2 t^{4}+t^{3}+2 t+4, t^{2}+3 t+1\right), \\
& \left(4 t^{8}+3 t^{7}+4 t^{5}+2 t^{4}+4 t^{3}+3 t+4, t^{2}+2 t+1\right), \\
& \left(4 t^{8}+4 t^{7}+t^{6}+3 t^{4}+t^{2}+4 t+4, t^{2}+t+1\right) .
\end{aligned}
$$

On the other hand, for a certain $S L\left(2 ; \mathbb{F}_{5}\right)$-representation $\rho_{0}$, the numerators and denominators of the twisted Alexander polynomials of $G\left(3_{1}\right)$ is given by

$$
\Delta_{3_{1}, \rho_{0}}^{N}(t)=t^{4}+2 t^{3}+2 t^{2}+2 t+1, \quad \Delta_{3_{1}, \rho_{0}}^{D}(t)=t^{2}+2 t+1 .
$$

For any $i$, it is seen that $\Delta_{8_{11}, \rho_{i}}^{N}(t)$ is not divisible by $\Delta_{3_{1}, \rho_{0}}^{N}(t)$ or $\Delta_{8_{11}, \rho_{i}}^{D}(t) \neq$ $\Delta_{3_{1}, \rho_{0}}^{D}(t)$. Then there exists no surjective homomorphism from $G\left(8_{11}\right)$ onto $G\left(3_{1}\right)$. Therefore we obtain

$$
8_{11} \nsucceq 33_{1} \text {. }
$$

Using Alexander polynomials, we cannot determine the non-existence of surjections between knot groups for 201 pairs of knots. However, we can prove the non-existence of surjections by using the twisted Alexander polynomials. To prove 
it, we take $2,3,5,7,11,17$ as a prime integer $p$ of $S L\left(2 ; \mathbb{F}_{p}\right)$-representation for $26,50,81,33,10,1$ cases respectively. All the data to check them are shown in [6].

\subsection{Construction of surjective homomorphisms}

In this subsection, we explain how to construct a surjective homomorphism between the groups of each pair of knots which appears in the list of Theorem 4.1.

Example 4.5 We show that there exist surjective homomorphisms $G\left(8_{5}\right) \rightarrow G\left(3_{1}\right)$ and $G\left(8_{18}\right) \rightarrow G\left(3_{1}\right)$. The knot group of $8_{5}, 8_{18}$ and $3_{1}$ admit Wirtinger presentations as follows:

$$
\begin{aligned}
& G\left(8_{5}\right)=\left(\begin{array}{c|c}
y_{1}, y_{2}, y_{3}, y_{4}, & y_{7} y_{2} \bar{y}_{7} \bar{y}_{1}, y_{8} y_{3} \bar{y}_{8} \bar{y}_{2}, y_{6} y_{4} \bar{y}_{6} \bar{y}_{3}, y_{1} y_{5} \bar{y}_{1} \bar{y}_{4}, \\
y_{5}, y_{6}, y_{7}, y_{8} & y_{3} y_{6} \bar{y}_{3} \bar{y}_{5}, y_{4} y_{7} \bar{y}_{4} \bar{y}_{6}, y_{2} y_{8} \bar{y}_{2} \bar{y}_{7}
\end{array}\right), \\
& G\left(8_{18}\right)=\left(\begin{array}{c|c}
y_{1}, y_{2}, y_{3}, y_{4}, & y_{4} y_{1} \bar{y}_{4} \bar{y}_{2}, y_{5} y_{3} \bar{y}_{5} \bar{y}_{2}, y_{6} y_{3} \bar{y}_{6} \bar{y}_{4}, y_{7} y_{5} \bar{y}_{7} \bar{y}_{4}, \\
y_{5}, y_{6}, y_{7}, y_{8} & y_{8} y_{5} \bar{y}_{8} \bar{y}_{6}, y_{1} y_{7} \bar{y}_{1} \bar{y}_{6}, y_{5} y_{8} \bar{y}_{5} \bar{y}_{7}
\end{array}\right), \\
& G\left(3_{1}\right)=\left\langle x_{1}, x_{2}, x_{3} \mid x_{3} x_{1} \bar{x}_{3} \bar{x}_{2}, x_{1} x_{2} \bar{x}_{1} \bar{x}_{3}\right\rangle,
\end{aligned}
$$

where $\bar{x}=x^{-1}, \bar{y}=y^{-1}$. We define a map $\varphi$ from $G\left(8_{5}\right)$ to $G\left(3_{1}\right)$ as follows:

$$
\begin{aligned}
& \varphi\left(y_{1}\right)=x_{3}, \varphi\left(y_{2}\right)=x_{2}, \varphi\left(y_{3}\right)=x_{1}, \varphi\left(y_{4}\right)=x_{3}, \\
& \varphi\left(y_{5}\right)=x_{3}, \varphi\left(y_{6}\right)=x_{2}, \varphi\left(y_{7}\right)=x_{1}, \varphi\left(y_{8}\right)=x_{3} .
\end{aligned}
$$

It is easy to check that this map $\varphi$ gives a homomorphism by computing the images of the relators. Moreover, it is clear that $\varphi$ is surjective by its definition. Then we obtain

$$
8_{5} \geq 31 \text {. }
$$

Next, we define another map $\varphi^{\prime}$ from $G\left(8_{18}\right)$ to $G\left(3_{1}\right)$ as follows:

$$
\begin{aligned}
& \varphi^{\prime}\left(y_{1}\right)=x_{1}, \quad \varphi^{\prime}\left(y_{2}\right)=x_{2}, \quad \varphi^{\prime}\left(y_{3}\right)=x_{1}, \varphi^{\prime}\left(y_{4}\right)=x_{3}, \\
& \varphi^{\prime}\left(y_{5}\right)=x_{3}, \varphi^{\prime}\left(y_{6}\right)=x_{1} x_{3} \bar{x}_{1}, \varphi^{\prime}\left(y_{7}\right)=x_{3}, \varphi^{\prime}\left(y_{8}\right)=x_{1} .
\end{aligned}
$$

Similarly it can be seen that the map $\varphi^{\prime}$ is also a surjective group homomorphism. Then we get

$$
8_{18} \geq 3_{1}
$$

In [6], we constructed surjective group homomorphisms for all pairs of knots in Theorem 4.1 explicitly. This completes the proof of Theorem 4.1.

We remark that we could find many surjective homomorphisms by using computer. Once they are found by computer, it is easy to check by hand that they are surjective homomorphisms. 


\section{Further results related to Theorem 4.1}

In the previous section, we determined the partial order " $\geq$ " in Rolfsen's knot table. These results lead to the following problems:

(1) Are three knots $3_{1}, 4_{1}$ and $5_{2}$ minimal elements?

(2) Which relation can a pair of a periodic knot and its quotient knot realize?

(3) Which relation can a degree one map between knot exteriors realize?

In this section, we give partial answers to these problems. First, we prove that $3_{1}$ and $4_{1}$ are minimal elements. To do this, we study a surjective homomorphism from a fibered knot to another one. Next, we determine which relation a pair of a periodic knot and its quotient knot realizes. Finally, we study which relation given in Theorem 4.1 can be induced by a degree one map. We do not know the complete answer of the realizing problem for a degree one map. Here we describe which surjection constructed in the previous section is realized by a degree one map.

\subsection{Surjection between fibered knots}

In this subsection, we prove the minimality of $3_{1}$ and $4_{1}$.

Theorem 5.1 $3_{1}$ and $4_{1}$ are minimal elements under this partial ordering.

To prove this theorem, we start to study a surjective homomorphism from a fibered knot group to another knot group. Let $K_{1}$ and $K_{2}$ be knots in $S^{3}$. We assume that $K_{1}$ is a fibered knot of genus $g_{1}$. Here we obtain the following.

Proposition 5.2 If there exists a surjective homomorphism $\varphi: G\left(K_{1}\right) \rightarrow G\left(K_{2}\right)$, then $K_{2}$ is also a fibered knot. Further the genus $g_{2}$ of $K_{2}$ is less than or equal to $g_{1}$.

Proof By the result of Neuwirth [13] and Stallings [17], the commutator subgroup $\left[G\left(K_{1}\right), G\left(K_{1}\right)\right]$ of $G\left(K_{1}\right)$ is a free group of rank $2 g_{1}$, because $K_{1}$ is a fibered knot of genus $g_{1}$. It is isomorphic to the fundamental group of its fiber surface. Restricting this surjection $\varphi$ on $\left[G\left(K_{1}\right), G\left(K_{1}\right)\right]$, we have a surjection

$$
\left.\varphi\right|_{\left[G\left(K_{1}\right), G\left(K_{1}\right)\right]}:\left[G\left(K_{1}\right), G\left(K_{1}\right)\right] \rightarrow\left[G\left(K_{2}\right), G\left(K_{2}\right)\right] .
$$

Here it is clear that $\left[G\left(K_{2}\right), G\left(K_{2}\right)\right]$ is also a finitely generated group, because it is the image of $\left[G\left(K_{1}\right), G\left(K_{1}\right)\right]$ by a surjection $\varphi$. Hence the commutator subgroup $\left[G\left(K_{2}\right), G\left(K_{2}\right)\right]$ of $G\left(K_{2}\right)$ is also a free group of a finite rank. By applying the result of [13] and [17] again, it is seen that $K_{2}$ is also a fibered knot. The genus of $K_{2}$ is denoted by $g_{2}$. Since $\varphi$ is a surjection between free groups, it is clear that $g_{2}$ is less than or equal to $g_{1}$. This completes the proof. 
As a corollary, we can prove Theorem 5.1 as follows.

Proof of Theorem 5.1 We put $K=3_{1}$ or $4_{1}$. They are the fibered knots of genus 1. Now we assume that $K^{\prime}$ is a non-trivial prime knot such that $K \geq K^{\prime}$. Here there exists a surjective homomorphism $\varphi: G(K) \rightarrow G\left(K^{\prime}\right)$ from the above assumption. By the above proposition, $K^{\prime}$ is a fibered knot of genus 1 , too. Since any genus 1 fibered knot is $3_{1}$ or $4_{1}, K^{\prime}$ is $3_{1}$ or $4_{1}$. It means that $3_{1}$ and $4_{1}$ are minimal elements.

We remark that Silver and Whitten studied the same result in [16, Proposition 3.11]

\subsection{Period of a knot}

The periods of knots with up to 10 crossings are listed in [7]. To supplement Theorem 4.1 , we have the following theorem.

Theorem 5.3 The following relations are realized by pairs of a periodic knot and its quotient knot:

$$
\begin{gathered}
8_{5}, 8_{15}, 8_{19}, 8_{21}, 9_{1}, 9_{16}, 9_{28}, 9_{40}, \\
10_{61}, 10_{63}, 10_{64}, 10_{66}, 10_{76}, 10_{78}, 10_{98}, 10_{139}, 10_{141}, 10_{142}, 10_{144} \geq 3_{1}, \\
8_{18}, 10_{58}, 10_{60}, 10_{122}, 10_{136}, 10_{138} \geq 4_{1}, \\
10_{120} \geq 5_{2} .
\end{gathered}
$$

\subsection{Degree one maps}

Earlier we constructed surjections to prove Theorem 4.1. In this subsection, we study which surjection is induced by a degree one map.

First, we recall the definition of a degree one map in the case of a knot exterior. Let $E\left(K_{i}\right)$ be oriented knot exteriors of $K_{i}$ in $S^{3}$ for $i=1,2$. A continuous map

$$
f:\left(E\left(K_{1}\right), \partial E\left(K_{1}\right)\right) \rightarrow\left(E\left(K_{2}\right), \partial E\left(K_{2}\right)\right)
$$

is called a degree one map if its induced map

$$
f_{*}: H_{3}\left(E\left(K_{1}\right), \partial E\left(K_{1}\right) ; \mathbb{Z}\right) \rightarrow H_{3}\left(E\left(K_{2}\right), \partial E\left(K_{2}\right) ; \mathbb{Z}\right)
$$

has a degree one. It is known that a degree one map $f:\left(E\left(K_{1}\right), \partial E\left(K_{1}\right)\right) \rightarrow$ $\left(E\left(K_{2}\right), \partial E\left(K_{2}\right)\right)$ induces a surjective homomorphism $f_{*}: G\left(K_{1}\right) \rightarrow G\left(K_{2}\right)$. See Hempel [2] for this fact. 
Let $f:\left(E\left(K_{1}\right), \partial E\left(K_{1}\right)\right) \rightarrow\left(E\left(K_{2}\right), \partial E\left(K_{2}\right)\right)$ be a degree one map. By the definition of degree one map, the induced map

$$
f_{*}: H_{3}\left(E\left(K_{1}\right), \partial E\left(K_{1}\right) ; \mathbb{Z}\right) \rightarrow H_{3}\left(E\left(K_{2}\right), \partial E\left(K_{2}\right) ; \mathbb{Z}\right)
$$

is an isomorphism. From the homology long exact sequence, we have

$$
0 \rightarrow H_{3}\left(E\left(K_{i}\right), \partial E\left(K_{i}\right) ; \mathbb{Z}\right) \stackrel{\partial}{\rightarrow} H_{2}\left(\partial E\left(K_{i}\right) ; \mathbb{Z}\right) \rightarrow H_{2}\left(E\left(K_{i}\right) ; \mathbb{Z}\right) \rightarrow \cdots
$$

Since each knot exterior $E\left(K_{i}\right)$ is homologically a circle, then $H_{2}\left(E\left(K_{i}\right) ; \mathbb{Z}\right)$ is vanishing. Hence the above boundary map

$$
\text { ว: } H_{3}\left(E\left(K_{i}\right), \partial E\left(K_{i}\right) ; \mathbb{Z}\right) \rightarrow H_{2}\left(\partial E\left(K_{i}\right) ; \mathbb{Z}\right)
$$

is an isomorphism. By the naturality, it is seen that

$$
f:\left(E\left(K_{1}\right), \partial E\left(K_{1}\right)\right) \rightarrow\left(E\left(K_{2}\right), \partial E\left(K_{2}\right)\right)
$$

is a degree one map if and only if $\left.f\right|_{\partial E\left(K_{1}\right)}: \partial E\left(K_{1}\right) \rightarrow \partial E\left(K_{2}\right)$ is a degree one map. Since $\partial E\left(K_{i}\right)$ is a 2-dimensional torus, the degree of $f$ is determined by the determinant of $f_{*}: H_{1}\left(\partial E\left(K_{1}\right) ; \mathbb{Z}\right) \cong \mathbb{Z} \oplus \mathbb{Z} \rightarrow H_{1}\left(\partial E\left(K_{2}\right) ; \mathbb{Z}\right) \cong \mathbb{Z} \oplus \mathbb{Z}$.

Now we fix a basis of $H_{1}\left(\partial E\left(K_{i}\right) ; \mathbb{Z}\right)$ so that the first element is the meridian $m_{i}$ of $K_{i}$ and the second one is a longitude $l_{i}$ of $K_{i}$. By such fixing bases of $H_{1}\left(\partial E\left(K_{i}\right) ; \mathbb{Z}\right)$, we can represent $f_{*}$ as a $2 \times 2$-matrix. The determinant of this matrix is the degree of $f: \partial E\left(K_{1}\right) \rightarrow \partial E\left(K_{2}\right)$. In our examples in [6], any $f_{*}$ maps the meridian of $K_{1}$ to that of $K_{2}$. Then we have to compute only the image $f_{*}\left(l_{1}\right)$, which can be written $f_{*}\left(l_{1}\right)=a m_{2}+b l_{2}$. Under this setting, $f_{*}$ on the boundary can represented by the matrix $\left(\begin{array}{ll}1 & a \\ 0 & b\end{array}\right)$. Hence, it is clear that $f$ is a degree one map if and only if $b= \pm 1$.

By using the above argument, we can check which surjective homomorphism in [6] is induced by a degree one map.

Example 5.4 Here we consider $G\left(8_{5}\right), G\left(8_{18}\right)$ and $G\left(3_{1}\right)$ of Example 4.5 . We check whether or not the surjective homomorphisms which are constructed in Example 4.5 are induced by a degree one map.

First, we check whether or not the map $\varphi: G\left(8_{5}\right) \rightarrow G\left(3_{1}\right)$ of Example 4.5 is induced by a degree one map. Pairs of the meridians and the longitudes of $G\left(8_{5}\right)$ and $G\left(3_{1}\right)$ with respect to the presentations of Example 4.5 are chosen as follows respectively:

$$
\left(y_{1}, y_{7} y_{8} y_{6} \bar{y}_{1} y_{3} y_{4} y_{2} \bar{y}_{5} \bar{y}_{1} \bar{y}_{1} \bar{y}_{1} \bar{y}_{1}\right), \quad\left(x_{3}, \bar{x}_{2} \bar{x}_{3} \bar{x}_{1} x_{3} x_{3} x_{3}\right) .
$$


Clearly, the image of the meridian of $G\left(8_{5}\right)$ under $\varphi$ is the meridian of $G\left(3_{1}\right)$. On the other hand, the image of the longitude of $G\left(8_{5}\right)$ under $\varphi$ is

$$
\begin{aligned}
\varphi\left(\text { longitude of } 8_{5}\right) & =\varphi\left(y_{7} y_{8} y_{6} \bar{y}_{1} y_{3} y_{4} y_{2} \bar{y}_{5} \bar{y}_{1} \bar{y}_{1} \bar{y}_{1} \bar{y}_{1}\right) \\
& =x_{1} x_{3} x_{2} \bar{x}_{3} x_{1} x_{3} x_{2} \bar{x}_{3} \bar{x}_{3} \bar{x}_{3} \bar{x}_{3} \bar{x}_{3} \\
& =\bar{x}_{3} \bar{x}_{3} \bar{x}_{3} x_{1} x_{3} x_{2} \bar{x}_{3} \bar{x}_{3} \bar{x}_{3} x_{1} x_{3} x_{2} .
\end{aligned}
$$

Then the image of the longitude of $8_{5}$ is equal to the $(-2)$ times of the longitude of $3_{1}$ in the first homology group. Then this surjective homomorphism $\varphi$ is not induced by a degree one map.

Next, we check whether or not the map $\varphi^{\prime}: G\left(8_{18}\right) \rightarrow G\left(3_{1}\right)$ of Example 4.5 is induced by a degree one map. Similarly, we fix pairs of the meridians and the longitudes of $G\left(8_{18}\right)$ and $G\left(3_{1}\right)$ with respect to the presentations of Example 4.5:

$$
\left(y_{1}, \bar{y}_{4} y_{5} \bar{y}_{6} y_{7} \bar{y}_{8} y_{1} \bar{y}_{2} y_{3}\right), \quad\left(x_{1}, \bar{x}_{3} \bar{x}_{1} \bar{x}_{2} x_{1} x_{1} x_{1}\right) .
$$

Similarly, the image of the meridian of $G\left(8_{18}\right)$ under $\varphi^{\prime}$ is the meridian of $G\left(3_{1}\right)$. Moreover, the image of the longitude of $G\left(8_{18}\right)$ under $\varphi^{\prime}$ is

$$
\begin{aligned}
\varphi^{\prime}\left(\text { longitude of } 8_{18}\right) & =\varphi^{\prime}\left(\bar{y}_{4} y_{5} \bar{y}_{6} y_{7} \bar{y}_{8} y_{1} \bar{y}_{2} y_{3}\right) \\
& =\bar{x}_{3} x_{3} x_{1} \bar{x}_{3} \bar{x}_{1} x_{3} \bar{x}_{1} x_{1} \bar{x}_{2} x_{1} \\
& =x_{1} \bar{x}_{3} \bar{x}_{1} x_{3} \bar{x}_{2} x_{1} .
\end{aligned}
$$

Then it is seen that the image of the longitude of $8_{18}$ is equal to the longitude of $3_{1}$. Therefore this surjective homomorphism $\varphi^{\prime}$ is induced by a degree one map.

We can check which surjection of Theorem 4.1 is induced by a degree one map. Finally, we obtain the following as the result.

Theorem 5.5 The following relations are realized by degree one maps:

$$
\begin{gathered}
8_{18}, 10_{5}, 10_{9}, 10_{32}, 10_{40}, 10_{103}, 10_{106}, 10_{112}, 10_{114}, 10_{159}, 10_{164} \geq 3_{1}, \\
9_{37}, 9_{40} \geq 4_{1}, \\
10_{74}, 10_{122} \geq 5_{2} .
\end{gathered}
$$

We do not know whether or not other relations are realized by degree one maps. 


\section{Problems}

In this section, we present other problems connected with the partial order $\geq$.

In Subsection 5.1, we proved that $3_{1}$ and $4_{1}$ are minimal. However, it remains open whether or not $5_{2}$ is minimal. So the first problem is the following.

(1) Characterize and determine the minimal knots under this partial order.

As we see in Subsection 5.1, if there exists a surjective homomorphism from the knot group of a fibered knot onto another knot group, then its target knot is fibered. We can restrict the partial order to the set of prime fibered knot.

(2) Determine the partial order on the set of fibered knots.

The next problem is to study the relation between this partial ordering and knot invariants. There are some invariants to measure and classify the complexity of knots. The most fundamental invariant is the crossing number. So the following problem arises naturally.

(3) If there exists a surjective homomorphism from $G\left(K_{1}\right)$ to $G\left(K_{2}\right)$, then is the crossing number of $K_{1}$ greater than that of $K_{2}$ ?

By Theorem 4.1, the answer is positive in case the crossing number is smaller than or equal to 10. Moreover, we can check that there exists no surjective homomorphism from the knot groups of knots with up to 10 crossings to that of alternating knots with 11 crossings. It still remains open for higher crossing cases.

The next problem also arises naturally.

(4) If there exists a surjective homomorphism from $G\left(K_{1}\right)$ to $G\left(K_{2}\right)$, then is the bridge number of $K_{1}$ greater than or equal to that of $K_{2}$ ?

The answer is also positive in case the crossing number is smaller than or equal to 10 .

(5) If there exists a surjective homomorphism from $G\left(K_{1}\right)$ to $G\left(K_{2}\right)$ and both of $K_{1}$ and $K_{2}$ are hyperbolic knots, then is the volume of $S^{3}-K_{1}$ greater than that of $S^{3}-K_{2}$ ?

We can calculate the hyperbolic volume for a given knot by SnapPea [19], or find its value on the web page KnotInfo [10]. Thus we can make sure that every pair of knots in the list of Theorem 4.1 satisfies this inequality.

In Subsection 5.3, we considered which surjective homomorphisms are induced by degree one map. However, this was restricted to the surjective homomorphisms which are constructed in [6]. We do not know whether or not other pairs $K_{1} \geq K_{2}$ can be realized by degree one maps. There may exist another surjective homomorphism other 
than we constructed, which is induced by degree one map. Therefore we should study the following problem.

(6) Decide all pairs of knot in Rolfsen's table which admit degree one maps.

We could not determine all geometric interpretations for the existences of surjective homomorphisms. However, Ohtsuki, Riley and Sakuma gave a systematic construction of surjective homomorphisms between 2-bridge link groups [14].

Acknowledgements The authors would like to express their thanks to Professors Sadayoshi Kojima and Dieter Kotschick for their useful comments and to Professor Makoto Sakuma for explaining to us results on periods of knots.

\section{References}

[1] R H Crowell, R H Fox, Introduction to knot theory, Graduate Texts in Mathematics 57, Springer, New York (1977) MR0445489 Reprint of the 1963 original

[2] J Hempel, 3-Manifolds, Ann. of Math. Studies 86, Princeton University Press (1976) MR0415619

[3] B J Jiang, S C Wang, Twisted topological invariants associated with representations, from: "Topics in knot theory (Erzurum, 1992)", NATO Adv. Sci. Inst. Ser. C Math. Phys. Sci. 399, Kluwer Acad. Publ., Dordrecht (1993) 211-227 MR1257911

[4] P Kirk, C Livingston, Twisted Alexander invariants, Reidemeister torsion, and CassonGordon invariants, Topology 38 (1999) 635-661 MR1670420

[5] T Kitano, Twisted Alexander polynomial and Reidemeister torsion, Pacific J. Math. 174 (1996) 431-442 MR1405595

[6] T Kitano, M Suzuki, A partial order in the knot table, Experiment. Math. 14 (2005) 385-390 MR2193401

[7] T Kitano, M Suzuki, A partial order in the knot table II, to appear in Acta Math. Sin. (Engl. Ser.)

[8] T Kitano, M Suzuki, M Wada, Twisted Alexander polynomials and surjectivity of a group homomorphism, Algebr. Geom. Topol. 5 (2005) 1315-1324 MR2171811

[9] X S Lin, Representations of knot groups and twisted Alexander polynomials, Acta Math. Sin. (Engl. Ser.) 17 (2001) 361-380 MR1852950

[10] C Livingston, JC Cha, Table of Knot Invariants Available at http:// www. indiana.edu/ knotinfo/

[11] J Milnor, A duality theorem for Reidemeister torsion, Ann. of Math. (2) 76 (1962) 137-147 MR0141115

[12] K Murasugi, Lectures at the Workshop in Osaka City University (December 2003) 
[13] L P Neuwirth, Knot groups, Annals of Mathematics Studies 56, Princeton University Press, Princeton, N.J. (1965) MR0176462

[14] T Ohtsuki, R Riley, M Sakuma, Epimorphisms between 2-bridge link groups, preprint (2006)

[15] D Rolfsen, Knots and links, Mathematics Lecture Series 7, Publish or Perish, Berkeley, CA (1976) MR0515288

[16] DS Silver, W Whitten, Knot group epimorphisms, J. Knot Theory Ramifications 15 (2006) 153-166 MR2207903

[17] J Stallings, On fibering certain 3-manifolds, from: "Topology of 3-manifolds and related topics (Proc. The Univ. of Georgia Institute, 1961)”, Prentice-Hall, Englewood Cliffs, N.J. (1962) 95-100 MR0158375

[18] M Wada, Twisted Alexander polynomial for finitely presentable groups, Topology 33 (1994) 241-256 MR1273784

[19] J Weeks, SnapPea Available at http://geometrygames.org/SnapPea/ index.html

Department of Information Systems Science, Faculty of Engineering

Soka University, Tokyo, 192-8577, Japan

Department of Mathematics, Akita University

Akita, 010-8502, Japan

kitano@soka.ac.jp, macky@math.akita-u.ac.jp

Received: 31 May 2006 Revised: 19 September 2007 
